\title{
THE MAIN LEITMOTIFS OF CHINESE MEDICINE IN THE CONTEXT OF THE DEVELOPMENT OF MODERN SCIENCE
}

D0I: $10.36740 /$ WLek202005130

\author{
Ihor A. Sniehyrov ${ }^{1}$, Inna A. Plakhtiienko ${ }^{2}$, Viktoriia 0. Kurhanska ${ }^{2}$, Yurii V. Smiianov ${ }^{2}$ \\ 'SUMY STATE A.S. MAKARENKO PEDAGOGICAL UNIVERSITY, SUMY, UKRAINE \\ 2SUMY STATE UNIVERSITY, SUMY, UKRAINE
}

\begin{abstract}
The aim: To demonstrate the limitations of pharmacological (chemical) therapy and the atomistic paradigm of science (in particular medicine) on the methodological basis of modern interdisciplinary directions (the theory of dissipative structures, chaos, autopoiesis), quantum mechanics, as well as the basic patterns of oriental medicine.

Materials and methods: The principles used in the article include self-organization, emergence, quantum mechanics (the Heisenberg uncertainty principle), the principle of consistency; principles of using coherent millimeter waves of low power, etc. Theoretical methods of analysis and synthesis, idealization, abstraction, induction and deduction are also used.

Conclusions: The concept of "integrable system" is equivalent to the concept of "integral quantum-mechanical system"; Integral quantum-mechanical systems (nuclei, atoms, molecules, living objects) in the ground state are described by periodic wave functions of the type exp (jwt); The traditional paradigm, for the most part, eliminates the qualitative difference between living and dead matter; Any living system functioning as a whole is simultaneously a macroscopic quantum-mechanical object and a millimeter-wave laser.
\end{abstract}

KEY WORDS: quantum mechanical system, biological coherence

Wiad Lek. 2020;73(5):1000-1003

\section{INTRODUCTION}

Despite the fact that the term "physics of the living" was somehow adopted by the scientific community, the boundaries of this new science are quite blurred, especially in the area of its contact with traditional biophysics. Therefore, when it comes to the fact that the physics of living is considered as the theoretical basis of quantum medicine - a fundamentally new area of modern medicine - this refers to the most radical direction of "physics of living", which is based on the idea that after nuclear, atomic and molecular, living is the fourth level of the quantum organization of nature.

There is a worldview, methodological and therapeutic dichotomy between the eastern and western approaches to solving the problem of restoring human health (and to the essence of life as a systemic whole). Western science, in particular medicine, is based on the classical paradigm with the predominance of an atomistic-mechanistic pattern in it. This means that the whole is treated as the sum of the elements with the resulting leveling of the principle of emergence. The results of microscopic examination are recognized as convincing, and the conclusions of macroscopic analysis in the best case scenario are considered as a preliminary stage preceding a thorough microscopic study.

Simultaneously, holism plays an essential role in the medicine of the East, within the framework of which man is organically inscribed in the Nature-Cosmos system. Using this approach, eastern medicine operates with categories that until recently were something alien to the European mentality and fell under the category of mysticism [1].

\section{THE AIM}

To demonstrate the limitations of pharmacological (chemical) therapy and the atomistic paradigm of science (in particular medicine) on the methodological basis of modern interdisciplinary directions (the theory of dis sipative structures, chaos, autopoiesis), quantum mechanics, as well as the basic patterns of oriental medicine.

\section{MATERIALS AND METHODS}

The principles used in the article include self-organization, emergence, quantum mechanics (the Heisenberg uncertainty principle), the principle of consistency; principles of using coherent millimeter waves of low power, etc. Theoretical methods of analysis and synthesis, idealization, abstraction, induction and deduction are also used.

\section{REVIEW AND DISCUSSION}

The atomistic aspect of the classical paradigm has given rise to pharmaceutical therapy (not unreasonably positive in its socio-historical context), which can be called "chemical therapy" due to the fact that its therapeutic effect is achieved due to the short-acting chemical effect on the 
sequence of chemical reactions in the subcellular structures of the living system. In this understanding, biochemical cycles and reaction chains are spatially localized and can be controlled during the experiment. Therefore, processes that are currently being described are not only occurring at the cellular and subcellular levels of a living organism, but in some cases, the functions of the organism are understood at these levels. An example is the decoding of the mechanism of chromosomal heredity in a sequential chain from nucleotides to amino acids and further to proteins $[2$, 3 ] and the accumulation of metabolic energy in the form of a charge on cell membranes via the proton transport mechanism [4].

However, the essence of the synchronization of processes in billions of body cells, the organization of differentiation of tissue cells, the implementation of gene information at the level of the whole body remains a mystery. These questions are the pinnacle of a more global and somewhat philosophical question: where is that line that separates living matter from dead matter? The answer at the descriptive level of Eastern philosophy or at the level of the tautological Engels definition "Life is a way of existence of protein bodies" [5] does not suit us within the framework of this article. Moreover, the conceptual-categorical apparatus must meet the requirements and criteria of the information paradigm of science.

It should also be noted that the "chemical force" is a type of electromagnetic force that occurs during the contact interaction of reagents, so it is a short-range component of the fundamental electromagnetic interaction. Under solid conditions, due to the screening effect, the long-range components of the electromagnetic interaction are leveled, so the microscopic approach is justified in these areas of traditional physics. But when studying wildlife, we are faced with the limitations of this method.

Indeed, in the process of systematic comprehension of the numerous experimental results of modern interdisciplinary research in the framework of the theory of dissipative structures, the theory of chaos, autopoiesis, etc., it becomes quite evident that considering short-range chemical forces as priority, in principle it is impossible to explain the potential of occurrence and existence during a relatively long time macroscopic forms of animal organisms. In other words, the traditional paradigm, for the most part, eliminates the qualitative difference between living and dead matter. G. Freilich [6] was one of the first who outlined the main contours of a new approach to this problem; he was looking for a source of the possibility of more effective long-range action in the plane of biological coherence research.

The formation of the concept of biological coherence began in the early 80 s of the last century in the context of revealing the natural characteristic frequencies of the human body [7]. The possibility of restoring the health status of the patient's body by exposure to low-intensity electromagnetic radiation, tuned in frequency in the millimeter range to human biologically active points (BAP), was demonstrated. In this context, it should be noted that
BAP coincide with acupuncture points, well known in ancient Chinese medicine. As studies in this direction have shown, this property is inherent to the living matter solely.

The experimental results [8] and, first of all, the vast clinical experience gained in various countries during the successful treatment of many thousands of patients suffering from "incurable" diseases from the point of view of conventional therapy, became the basis for understanding the leading role of coherent electromagnetic waves of the body in the realization of its genetic integrity and ensuring the diverse differential stability of the living. Awareness of this fact made it possible to formulate a hypothesis about the place of the living on the Weisskopf quantum staircase of nature.

Briefly, this hypothesis can be formulated as follows. Any living system functioning as a whole is simultaneously a macroscopic quantum-mechanical object and a millimeter-wave laser. It is proved that just such an approach to the problem gives a consistent explanation of the entire volume of experimental data, including the treatment of "incurable" diseases with the help of a few quanta of electromagnetic radiation. The formation of this idea was based on the recognition of the fact that the basic principles of quantum mechanics - the principles of identity and discreteness - and only they determine the diverse differential stability of the world at three levels of its quantum organization. We are talking about the three steps of the Weisskopf quantum ladder - nuclear, atomic and molecular - and in accordance with them there are three sections of fundamental physics. And the statement, according to which the diverse differential stability of living things (the existence of species and individual characteristics of plants and animals) should have the same reason, representing quantum-mechanical entities, is quite justified.

The problem of the reality of the quantum-mechanical integrity of a macroscopic object is closely related to the possibility of the existence of a unitary nonlocal self-consistent potential in it. Under ordinary conditions of a solid body, the presence of such a potential is impossible. That is why macroscopic physics, as a rule, solely deals with quantum effects, when extraordinary conditions are created, such as, for example, superconductivity, superfluidity, inverse population (laser mode), plasma state, etc.

What the situation in the field of living matter is like?

First of all, it is necessary to recall the existence of a strong electric field on the protoplasmic membranes of every living cell. Considering that the physical properties of membranes make it possible to oscillate at natural frequencies in the millimeter wavelength range under vacuum conditions, we can conclude that any cell of any living organism can be considered as an active center, therefore, to regard it as a source of electromagnetic pump radiation. Despite this, in most cases, it cannot be argued in advance that the existence of active centers in the medium is a sufficient condition for the transition to laser generation. Very strong absorption in the tissues can impede the passage of the system through the threshold of a nonequilibrium phase transition. And that is where we should turn to the ideas of oriental medicine. 
The essence of the matter is that the BAPs are not located on the surface of the human body arbitrarily, they are organized in such a way that most of them are located on specific lines called meridians [9], more precisely, on the external tracks of these meridians, since in addition there are internal Meridian tracks passing through the main organs of the body, in accordance with the ideas of Eastern philosophy. Thus, the whole body is penetrated by 28 meridians, and it may seem that the mechanism of action of acupuncture is easy to understand. But not from the point of view of Western medicine, since there are no morphological features associated with the trajectories of the meridians, at the same time, it is morphologically visualized in the tradition of Western medicine.

The Rudenko effect (observation of external trajectories of meridians with the help of weak magnetic fields comparable with the intensity of the Earth's field) showed the limitations of this point of view and demonstrated the electromagnetic nature of the Chinese meridians. This fact, along with the stability of the trajectories of the meridians, as well as the gauge invariance of their lengths with respect to the distances between the main anatomical features of the body, made it possible to look at these trajectories as projections in three-dimensional space of phase trajectories of stable coherent medium waves (limit cycles).

In this context, the presence of a meridian is sufficient evidence of the existence of a nonlocal, self-consistent electromagnetic potential of the body as a whole and, therefore, the existence of effective long-range forces that ensure the quantum-mechanical integrity of the body beyond the threshold of a nonequilibrium phase transition.

Thus, limit cycles can be considered as a reflection on the phase plane of a stable solution of the simplest nonlinear differential equation used in synergetics in the case of the one-dimensional Landau-Haken potential. As is known, after conversion to a two-dimensional structure this potential can be visually presented in the form of a model similar to a bottle turned upside down with a steady movement of the "probe" along the ramp along a circular path simulating an electromagnetic limit cycle.

This synergistic form of quantum-mechanical potential, observed in this situation, allows us to generalize this result to the problems of traditional quantum mechanics. It also stimulated the replacement of commonly used forms of potential wells, which gave a solution in the form of stable waves, synergistic potentials with solutions in the form of traveling waves. This replacement gives physical meaning to the mechanism of quantization of energy levels, regenerating in this way the intuitive prediction made by N. Bohr [10]. Running coherent electromagnetic waves are formed in the human body and propagate within it in accordance with the known laws of nonlinear optics for a closed resonator filled with a nonlinearly absorbing medium with active centers. According to the principle of minimal losses, the aforementioned waves must fall on the skin surface from the inside at an angle greater than the angle of total internal reflection for a given medium. An analysis of the localization of acupuncture points along the outer trajectories of the meridians confirmed this assumption. The dimensions of the cross sections of the meridians are determined by the condition of saturation of the medium along their tracks and in most cases, the exact formation of the latter can be carried out by reflecting electromagnetic waves from the nails of the fingers and toes.

The next problem is the informational content of the spectrum of traveling electromagnetic waves that form meridians in its connection with the genome and the properties of a holistic quantum system. It is known that all somatic cells of the body have the same genome. It is this property that makes it possible to consider any cell as an active center of the coherent field of an organism. To transfer the information contained in the genes, this field must receive this information from DNA. In this context, one cannot disagree with S. Webb's point of view that intracellular processes are synchronized by an internal coherent electromagnetic field. We can say that the translation of chemical information into an electromagnetic language, and vice versa, can be carried out through the mechanism of laser chemical pumping (Polanyi) during decryption in the following sequence: DNA - microtubules (cytoskeletal network) - membranes [11]. It can also be assumed that the cell membrane plays the role of a high-Q active filter with a bandwidth in the millimeter range (due to its shape and charge) and is controlled by the membrane channel system. This allows to eliminate the exit from the cells of the high-frequency part of the spectrum, which carries out self-consistent communication during intracellular processes.

Based on the above concept, there is no direct connection between one or another gene and the morphological specificity of the organism. Genes encode the formation of molecules (as it is known) and form the features of a self-consistent potential, thus creating a "rule" of selection that transforms the quasi-continuous spectrum of energy transitions into a discrete one that is unique to this particular organism. Thus, the formation of the spectrum of characteristic eigenfrequencies occurs. This spectrum determines all the morphological properties of the species, specific to each creature, similar to how it happens on other steps of the quantum ladder. Thus, the spectra of natural characteristic frequencies are, in fact, universal passports of stable integrity for objects of both inanimate and living nature.

In this context, the problem comes to the forefront, causing widespread discussion in monographs and on the pages of scientific journals, when the ideas of the theory of self-organization, chaos revived interest in the problems of time and its reversibility, entropy, integrable and non-integrable systems, dynamic chaos, etc.

When trying to clarify the nature of the irreversibility of time, the imaginary sign $j$ usually refers to the energy frequency [12]. The temporary variable $t$ refers to the imaginary sign $\mathrm{j}$. It means that in the ground state, integral quantum mechanical systems do not have real time. For example, we cannot apply the concept of time to a hydrogen atom in the ground state (what is the age of this hydrogen 
atom?), despite the fact that it exists in the world with current time. And only with external influence (transition to an excited state, radioactivity) does the real part of the complex time appear: the lifetime in the excited state, the half-life in radioactivity.

However, if particles or quantum integrity collide, form a new integral quantum-mechanical system, then the real part of the complex time disappears, and the system begins to be described by a periodic wave function with imaginary time. In the language of classical physics, this means that we entered the regime of an integrable system and can exclude potential energy from the Hamilton operator (and, therefore, from the total energy), that is, we can perform the canonical transformation and show that in the new coordinates (the angle is variable action) there is no interaction of the integrable system with the rest of the world. The integrable system depends on only one variable - the action, and therefore, by analogy with the integral quantum system, it is described by only one wave function.

Questions of the theory of development in general and embryology in particular are also in the sphere of interests of living physics. Now we are trying to understand the mechanism of formation of the «electromagnetic framework» of the embryo in its self-consistency with the biochemical development of the child. It should be noted that the ideas of living physics do not contradict the existence of a biochemical picture of the development of living things. On the contrary, the ideas expressed above fill the gaps in this picture.

\section{CONCLUSIONS}

Based on the concept of living physics, we can draw the following conclusions in this vein:

- firstly, the concept of "integrable system" is equivalent to the concept of "an integral quantum-mechanical system"; - secondly, integral quantum-mechanical systems (nuclei, atoms, molecules, living objects) in the ground state are described by periodic wave functions of the exp type (jwt). - thirdly, BAP coincide with acupuncture points, well known in ancient Chinese medicine. As further studies in this direction have shown, this property is inherent solely to the living matter.

The World Health Organization has repeatedly expressed concern about the abuse of developed countries by pharmacological therapy. In particular, the potential vulnerability of mankind to pneumonia and tuberculosis was pointed out. In this context, we would like to conclude with a few paraphrases of Claude Levy-Strauss: "The 21st century will be a century of quantum medicine - or it won't be at all."

\section{REFERENCES}

1. Kapra F. Dao fiziki. [Physics' Dao]. Kiev: «Sofiya», Moscow: ID «Gelios», 2017; 22-32. (Ru).

2. Crick F. H. On protein synthesis. Symp. Soc. Exp. Biol. 1958; 12:138-163.

3. Uotson D. Dvoynaya spiral. [Double Spiral]. Moscow: AST. 2019: 256. (Ru)

4. Nelson, David; Cox, Michael. Lehninger Principles of Biochemistry. New York:W.H. Freeman. 2013: 403.

5. Engels F. Anti-Dyuring. Dialektika prirodyi. [Nature Dialectics]. Moscow: AST. 2016; 542 (Ru).

6. Frohlich $\mathrm{H}$. Long range coherence and energy storage in biological systems. Inf. Of Quantum Chem. 1968; 2: 641-649.

7. Andreev E. A., Belyiy M. U. Proyavlenie sobstvennyih harakternyih chastot chelovecheskogo organizma. [Expression of the human being's body characteristic features]. Progress. 1989; 34-36 (Ru).

8. Sitko S.P. Mkrtchan L. N. Vvedenie vkvantovuyu meditsinu. [Introducton into kvant medicine]. Kiev: Pattern. 2019; 56-64 (Ru).

9. Kapra F. Dao fiziki. [Physics'Dao]. Kiev: «Sofiya», M.: ID «Gelios». 2017; 76-82 (Ru).

10. Kuznetsov B. G. Printsip dopolnitelnosti. [Complementarity Principle]. Moscow: URSS. 2016; 34-36 (Ru).

11. Koruga D. L. Neurocomputing in book. Ed. E.Pelikan, N. Novak. 2009; 19-39.

12. Prigozhin I., Stengers I. Vremya, haos, kvant. [Time, Chaos, Quant]. Moscow: URSS. 2019; 64-66. (Ru).

Research topic of the Department of Philosophy and Social Sciences "Strategy of development of Ukraine as a social system under the influence of science-intensive technologies (nonlinearity constancy)" for 2020-2022. State registration number: 0120U100571.

\section{ORCID and contributionship:}

Ihor A. Sniehyrov - 0000-0002-2624-2097 A,D,F

Inna A. Plakhtiienko - 0000-0002-4200-8997 ${ }^{B}$

Viktoriia O. Kurhanska - 0000-0003-3250-2112 E,F

Yurii V. Smiianov - 0000-0002-3311-4210 E,F

\section{Conflict of interest:}

The Authors declare no conflict of interest

\section{CORRESPONDING AUTHOR Ihor A. Sniehyrov \\ G. Kondratieva Str. 165, building 148, apt. 14, 40021, Sumy, Ukraine \\ tel: +380508329319 \\ e-mail: snegilecter@rambler.ru}

Received: 26.01 .2020

Accepted: 01.04 .2020

A - Work concept and design, B - Data collection and analysis, C - Responsibility for statistical analysis, D-Writing the article, $\mathbf{E}$ - Critical review, $\mathbf{F}$ - Final approval of the article 